\title{
Portfolio Sales and Signaling $\ddagger$
}

\author{
Spiros Bougheas ${ }^{\mathrm{a}, *}$, Tim Worrall ${ }^{\mathrm{b}, 1}$ \\ ${ }^{a}$ School of Economics, University of Nottingham, University Park, NG7 2RD, United Kingdom. \\ ${ }^{b}$ School of Economics, University of Edinburgh, 31 Buccleuch Place, EH8 9JT, United Kingdom.
}

\begin{abstract}
This paper extends the DeMarzo and Duffie (1999) signaling model from single sales to portfolio sales. It shows that the extended model can account for retention of low quality assets and help explain why retained assets may be of varying quality.

Keywords: Securitization, Signaling, Skin in the game

JEL: D82, G21, G23
\end{abstract}

\footnotetext{
${ }^{\ddagger}$ We would like to thank the editor and referees as well as Daniel Seidmann, Javier Suarez and Tianxi Wang for many helpful comments and suggestions. The usual disclaimers apply.

* Corresponding author.

Email addresses: spiros.bougheas@nottingham.ac.uk (Spiros Bougheas), tim.worrall@ed.ac.uk (Tim Worrall)

${ }^{1}$ The second author would like to acknowledge the support of ESRC grant ES/L009633/1.
} 


\section{Introduction}

It is widely recognized that, in the presence of asymmetric information, banks may retain a proportion of the securities they sell in order to signal asset quality. That is, they keep a 'skin in the game'. DeMarzo and Duffie (1999) provide a model to show how this skin in the game is inversely related to asset quality and that the lowest quality asset is not retained. A number of studies (see, for example, Chen et al., 2008; Demiroglu and James, 2012) provide empirical evidence supporting the prediction of an inverse relation between retention and asset quality. On the other hand, Acharya et al. (2009) provides contradictory evidence that assets with the highest risk (the lowest quality) are nearly always retained.

Another implication of the DeMarzo and Duffie (1999) model is that securities sold will be homogeneous in terms of quality. In particular, DeMarzo (2005) demonstrates that when sellers have better information about assets than potential buyers, they are better off selling them separately rather than pooling them together. The intuition is that pooling reveals no information to buyers - it destroys information, and therefore cannot be beneficial for sellers. The empirical support for this prediction is mixed. Gorton and Metrick (2013) find that securities sold are broadly of homogeneous quality. Keys et al. (2010), on the contrary, suggest that within broad categories of risk, there is considerable heterogeneity of quality within the securities sold.

The purpose of this paper is to extend the DeMarzo and Duffie (1999) model to explain why assets of low quality might be retained and why the retained securities may involve assets of varying quality. We do this by allowing for multiple assets and allowing banks to sell securities as portfolios. We show that as long as banks can commit to the menu of contracts that they offer to investors, or commit to sell their whole portfolio of assets, then there are parameter values such that portfolio sales dominate single asset sales and the securities sold can be of varying and low quality.

We present the model in Section 2. We consider a bank whose assets are a portfolio of two loans. Loans are sold to investors in a competitive market. All agents are risk neutral and therefore, prices are equal to the conditional expected value. Each loan can be either high-quality (high probability of repayment) or low-quality. Returns on the two loans are assumed to be independently distributed. With two loans and two loan types, 
there are three possible portfolio types: both loans are high quality (a portfolio we label as $\mathrm{H}$ ), one is high quality and the other is low quality (M), or both are low quality (L). As in Shleifer and Vishny (2010), a bank makes profits by collecting fees when offering loans for sale. The funds the bank obtains from these sales are reinvested in taking on new loans. There is asymmetric information between sellers and buyers: the bank knows the quality of each loan on its books but investors cannot observe loan quality at the time of sale (the bank does not know the loan quality before bringing loans onto its books). Since there is asymmetric information, the bank may wish to signal the quality of loans by retaining a fraction of the loan on its books along the lines suggested by DeMarzo and Duffie (1999).

In Section 3 we replicate a result of DeMarzo (2005) that, other things being equal, banks are better off selling loans separately rather than selling them together as a portfolio (see Proposition 1). The intuition for this result is straightforward. Selling loans singly requires only one signal: that a loan is high quality. Selling loans as a portfolio requires two signals to distinguish the three portfolio types. Since signaling is costly, it is preferable to sell loans singly. Although this result can be found in DeMarzo (2005) and is straightforward, we present it in detail because it aids the understanding of subsequent results.

Section 4 extends the analysis by allowing banks to pool together a mix of two of the three possible portfolios. It presents our main result (Theorem 1) that describes when pooling a mix of two portfolios is optimal. ${ }^{2}$ The advantage of pooling two portfolios is that it requires only one signal, reducing signaling costs compared to signaling three types of portfolio. It may also be preferable to selling loans singly. For example, pooling together portfolios $\mathrm{M}$ and L involves a signal only if both loans are of high quality, whereas selling the loans singly requires a signal even when only one of the loans is of high quality. There is however, a disadvantage for the bank in pooling a mix of portfolios. Using a mix of portfolios changes the incentive constraints that must be satisfied for a signaling strategy to be credible. This may increase the cost of the required signal. We show that if the fee is high, then the bank will prefer to sell the loans together. If the fee is low, then the

\footnotetext{
${ }^{2}$ Note that pooling here is used in the sense of game theory and does not mean that the bank is creating a new security.
} 
bank prefers to retain the high quality loan than to mix portfolios and, thus, it will sell loans singly. For intermediate values of the fee, the bank finds it optimal to pool a mix of the portfolios $\mathrm{M}$ and $\mathrm{L}$ or a mix of the portfolios $\mathrm{H}$ and $\mathrm{L}$.

It may seem odd that a bank may wish to pool portfolios $\mathrm{H}$ and L. However, the skin in game increases with the difference in the quality between the two assets. The difference between the expected payoff from pooling the two portfolios $\mathrm{H}$ (two high quality loans) and L (two two quality loans) compared with the expected payoff from portfolio M (one low quality and one high quality loan) is relatively small. Thus, the bank can signal the difference by retaining only a small fraction of the pooled portfolio. When this is optimal, the fraction retained by the bank may include low quality loans. Taking an average over banks, the retained asset will include both high and low quality loans. Theorem 1 describes parameter values for which this is indeed optimal.

In addition to accounting for some observed retention strategies that are unexplained by DeMarzo and Duffie (1999), our model also delivers a novel prediction about how retention strategies change in response to uncertainty. During periods of increased uncertainty, it predicts that banks are more likely to use pooling strategies. That is, securities sold are more heterogeneous in periods of increased uncertainty. In Section 4, we explain in more detail the derivation of this prediction and suggest how it might be empirically tested.

Our paper is closely related to other work on signaling that builds upon the seminal work of Leland and Pyle (1977). This literature includes DeMarzo and Duffie (1999) and DeMarzo (2005) mentioned above. DeMarzo (2005) starts from a similar premise to our paper, namely that in the DeMarzo and Duffie (1999) model, banks prefer to sell securities singly rather than as portfolios. DeMarzo (2005) shows that banks may benefit from designing new securities that pool assets into tranches. In particular, banks can tranche securities into a risk-free, senior security and a residual 'equity' claim where all the default risk is concentrated. This strategy has the advantage that it ameliorates the lemons problem for the risk-free tranche, enabling banks to sell loans at a higher average price. In our model, there are only two states and in the bad state both loans return nothing. With this assumption, it is not possible to tranche assets in the way done by DeMarzo (2005). The DeMarzo and Duffie (1999) model has also been extended to a 
dynamic environment by Hartman-Glaser (2017) (see also Pagès, 2013). Hartman-Glaser (2017) shows that banks build a reputation over time that mitigates the power of retention as a signaling device. Although our main result applies to the single-period model, we discuss how it might be extended to a multiple-period context in Section 5. Our model does require some form of commitment (either to a menu of contracts or to stand ready to sell the whole portfolio) by the seller. We are not the first to use commitment to analyze securitization and portfolio sales. Gorton and Souleles (2007) argue that the willingness of banks to subsidize special purpose vehicles by buying back low quality assets, a type of 'implicit recourse', can be interpreted as a form of commitment. In our model, the commitment of banks to a menu of contracts plays a similar role.

We organize the paper as follows. Section 2 describes the model. Section 3 derives a preliminary result, similar to DeMarzo (2005), that compares single asset sales and portfolio sales. The main result of the paper is derived in Section 4. Section 5 considers how the model might be extended to multiple rounds of sales and Section 6 analyzes an alternative form of commitment. We offer some final comments in Section 7. Proofs not given in the text are relegated to the Appendix.

\section{The Model}

The economy lasts for one period and consists of banks and investors. All agents are risk-neutral. Banks are identical and we can focus the analysis on the behavior of a single bank. At the beginning of the period, the bank offers loans of unit size to finance two risky projects. There are two types of loans. Loans of type $j \in\{h, l\}$ repay $R$ with probability $\pi_{j}$ and fail to repay anything with probability $1-\pi_{j}$; where $\pi_{h}>\pi_{l}$. We assume that both types of loans have positive net present value; $\beta \pi_{l} R>1$, where the discount factor $\beta$ captures the time preference, common to all agents. Let $\theta$ denote the probability that a loan is of type $h$. Loan types are independently distributed. Let $\psi:=\theta \pi_{h}+(1-\theta) \pi_{l}$ be the unconditional probability that a loan repays $R$. The unconditional probability is relevant both for investors, who do not know the loan type, and for the bank when taking new loans onto its books. With two loans there are three portfolio types. With

probability $\theta^{2}$ both loans are of type $h$ (portfolio $H$ ); with probability $(1-\theta)^{2}$ both loans are of type $l$ (portfolio $L$ ) and with probability $2 \theta(1-\theta)$ one of the loans is of type $h$ and 
the other loan is of type $l$ (portfolio $M$ ). The probability $\theta$ and the size of the bank's portfolio are common knowledge to the bank and investors.

We assume that at the beginning of the period, before the bank learns its portfolio type, it commits to a set of contracts for selling its portfolio to investors. We will show that this commitment maximizes the bank's ex ante payoff, allowing it to use a pooling sales strategy. Commitment is required because such strategies will not necessarily maximize the bank's ex post payoff once it has learned its portfolio type. We will demonstrate that this commitment can explain the banks sells its loans as portfolios but do not necessarily pool together all loans. After the announcement of sales contracts, the bank learns the types of its loans (and, as a consequence, the type of its portfolio). ${ }^{3}$ This information is private. At this point, the bank can either keep the loans on its books or it can try to sell them to investors. This market for loans is assumed to be competitive. If sold, the bank can finance new loans. The bank collects a fee $f$ when it signs a new loan agreement. The role of the fee, as in Shleifer and Vishny (2010), is as a devise to generate trade between the bank and outside investors at the start of the trading period. The bank can also choose between selling the whole portfolio and single loan sales. When the bank keeps a loan on its books, it has to wait until the end of the period to receive a payoff. In contrast, when the bank sells the loan and uses the proceeds to make new loans it collects fees at the beginning of the period and purchases a new loan of unknown type. Define $\phi:=f+\beta \psi R$ to be the expected discounted payoff the bank anticipates from selling a unit of loan: the arrangement fee from the new loan plus the expected discounted return from the new loan. By assumption $\phi>1$. Investors observe the size of the bank's portfolio and its retention strategies but observe neither loan nor portfolio types.

Since investors do not observe the type of a loan, there is a lemons problem. The maximum amount that investors are willing to pay for a loan is $\beta \pi_{l} R$ because if they offer to pay $\beta \psi R$ (the expected loan payoff), then the bank will only sell $l$ type loans. Since keeping a loan on its books is costly in terms of the fees forgone, the bank might be able

\footnotetext{
${ }^{3}$ The lag between the announcement and the learning of types captures the period during which the bank learns the type of its portfolio (prime, sub-prime, etc.). The assumption that the bank knows the type of loans on its books after purchase but does not know the loan type at the time it brings loans onto its books is clearly an extreme one. It is however, meant to capture the idea that the bank has better information after it has kept a loan on its books for a while.
} 
to use a retention strategy, that is, keeping a fraction of a loan on its books as skin in the game, to signal the quality of the loan to investors. DeMarzo and Duffie (1999) show that the skin in the game, $d^{q}$, for a given asset quality $q$, is given by

$$
d^{q}=\frac{\phi\left(\frac{p_{q}}{p_{q^{-}}}-1\right)+d^{q^{-}}(\phi-1)}{\phi\left(\frac{p_{q}}{p_{q^{-}}}\right)-1}
$$

where $q^{-}$denotes the quality one level below $q$, and $p_{q}$ and $p_{q^{-}}$denote the competitive prices of the corresponding assets. Furthermore, when $q^{-}$is the lowest quality level, then $d^{q^{-}}=0$ : the lowest quality is not retained. The equation will be explained more fully in the next section. Essentially, the higher the quality of the loan, the lower is the cost of retaining it on the books. Therefore, banks signal quality by keeping a skin in the game that is inversely related to loan quality.

\section{Single Loans and Portfolio Sales}

In this section, we compare single loan sales and portfolio sales. We present a key benchmark result that in the absence of commitment, single loan sales dominate. This result was established by DeMarzo (2005). Given that our set-up is different from DeMarzo (2005), we go through the analysis in detail because it will help to understand the mixed pooling and signaling case presented in Section 4.

\subsection{Single Loan Sales}

In this subsection, we assume that the bank sells loans singly. An equilibrium may be either pooling or separating. First, consider a separating equilibrium. Let $d^{j}$ denote the fraction of a loan of type $j$ that the bank is supposed to keep on its books and let $p_{j}$ denote the price of a loan of type $j$. The profit to the bank with a loan of type $i$ of retaining a fraction $d^{j}$ of the loan on its books is:

$$
U_{i j}:=d^{j} \beta \pi_{i} R+\left(1-d^{j}\right) p_{j} \phi .
$$


The first term is the bank's expected payoff from keeping on its books a fraction $d^{j}$ of the loan that has a success probability of $\pi_{i}$. The second term is the sales revenues $\left(1-d^{j}\right) p_{j}$ from the fraction of the loan not retained times the expected discounted payoff the bank anticipates from selling a unit of loan $\phi=f+\psi \beta R$ : that is, the arrangement fee $f$ from the new loan plus the expected discounted return from the new loan $\psi \beta R$. Here the expected repayment probability of new loans is $\psi$ because at the point of purchase the bank is uncertain about the loan type.

The maximum price that an investor will pay for a loan of type $j$ is $\beta \pi_{j} R$ : that is, the loan's discounted expected payoff. Given that the market for loans is competitive, the price of loans will be bid up to this maximum value:

$$
p_{j}=\beta \pi_{j} R \quad \text { for each } j \text {. }
$$

Lemma 1. Under a separating equilibrium of prices and retention strategies with single loan sales:

$$
\begin{aligned}
p_{l}=\beta \pi_{l} R, \quad p_{h}=\beta \pi_{h} R, \\
d^{l}=0, \quad d^{h}=\hat{d}^{h}:=\frac{\phi\left(\frac{p_{h}}{p_{l}}-1\right)}{\phi\left(\frac{p_{h}}{p_{l}}\right)-1}=\frac{\phi\left(\pi_{h}-\pi_{l}\right)}{\phi \pi_{h}-\pi_{l}} .
\end{aligned}
$$

The expression for $d^{h}$ in equation (3) is the skin in the game retained by the bank to signal that the loan is high quality. Since $\phi>1$ and $\pi_{h}>\pi_{l}, d^{h} \in(0,1)$. The intuition for the result is quite straightforward. Since $\phi>1$ and the price received is given by (2), the bank will prefer, ceteris paribus, to sell a loan rather than retain it. With two loans each of two types and, hence, three portfolio types, there are six incentive constraints to ensure that the payoff from selling the loans according to the true portfolio type is no less than the payoff from selling the loans as one of the other two portfolio types. Despite there being six incentive constraints, it can be shown that the two relevant constraints are that the bank with portfolio $\mathrm{H}$ (two loans of type $h$ ) prefers not to sell it as a portfolio $\mathrm{L}$ (two loans of type $l$ ) and vice-versa. If these two constraints are satisfied, then so are all the others (see the appendix for the full proof). This means that the analysis of the single loans case is identical to the case where the bank has only one loan that can be of 
either high or low type. First, it is clear that there is no advantage to have $d^{l}>0$ because signaling is costly. Therefore, for the bank selling all of its low type loan, its expected payoff is $U_{l l}=p_{l} \phi$. Whereas, if it retains a fraction $d^{h}$ and receives a price $p_{h}=\beta \pi_{h} R$ for the loans sold, its expected payoff is $U_{l h}=d^{h} \beta \pi_{l} R+\left(1-d^{h}\right) p_{h} \phi=d^{h} p_{l}+\left(1-d^{h}\right) p_{h} \phi$, since the value of the loans retained is $p_{l}$. Incentive compatibility requires both $U_{l l} \geq U_{l h}$ and $U_{h h} \geq U_{h l}$. Combining these two conditions gives

$$
d^{h} p_{h} \geq\left(p_{l}-\left(1-d^{h}\right) p_{h}\right) \phi \geq d^{h} p_{l}
$$

Equivalently,

$$
\frac{\left(p_{h}-p_{l}\right) \phi}{p_{h}(\phi-1)} \geq d_{h} \geq \frac{\left(p_{h}-p_{l}\right) \phi}{p_{h} \phi-p_{l}} .
$$

It is clear that the most relevant constraint is that the bank should not wish to sell a low type loan as a high type. This is the second inequality in (4). Where it is satisfied as equality $\left(U_{l l}=U_{l h}\right)$, the value of $d^{h}$ is given by equation (3) in Lemma 1. An increase in $\phi$ reduces the required retention rate $\hat{d}^{h}$ because the cost of signaling is increased. A rise in the ratio of $\pi_{h} / \pi_{l}$ has the opposite effect because it makes passing off low quality loans as high quality more tempting and therefore the required retention rate to signal high quality increases.

In principle, any prices satisfying (2) and $d^{h}$ satisfying (4) can be supported as a separating equilibrium with investor beliefs that retention $d<d^{h}$ corresponds to a low quality loan and any $d \geq d^{h}$ comes from a high quality loan. However, as is standard, the separating equilibrium of Lemma 1 is the Pareto-dominant separating equilibrium that also satisfies the intuitive criterion. To see this, suppose that $d^{h}$ satisfies (4) and $d^{h}>\hat{d}^{h}$. A bank with a low type loan will never choose such a $d^{h}$. Thus, investors believe that any such deviation to a lower $d^{h}$ must come from a high type and therefore can be sold for the high price $p^{h}$. Given that the bank's payoff decreases with $d^{h}$, the amount retained will be decreased until $d^{h}=\hat{d}^{h}$.

Now, we consider the possibility of a pooling equilibrium. This requires that the bank has some form of commitment to a selling strategy before it knows its portfolio type. We suppose that the bank can commit to a menu of contracts conditional on sales taking place. We view the commitment as a short cut to modeling repeated interactions 
that might generate similar effects endogenously. We will consider a model with repeated interactions in Section 5. In Section 6, we will consider an alternative form of commitment (which will turn out to be a stronger form of commitment) in which the bank commits to a menu of contracts with each investor separately, but also commits to stand ready to sell all its loans. In a pooling equilibrium, the bank will not keep any fraction of the loan on its books given that retention is only beneficial if it can be used as a signal. Competition among investors means that in this case the price of loan sales is bid up to $\beta \psi R$. For this to be an equilibrium, the bank must prefer to sell its loans rather than retaining them. For a loan of type $l$, the bank's expected payoff from sales is $\phi \beta \psi R$ compared with $\beta \pi_{l} R$ from retention. Since $\phi>1$ and $\psi>\pi_{l}$, it follows that sales are always better than retention. On the other hand, if the bank sells a high quality loan to investors, its payoff will be $\phi \beta \psi R$ compared to $\beta \pi_{h} R$ from retention. Therefore, if $\phi \psi<\pi_{h}$ the bank would prefer to keep the high quality loan on its books rather than earning the pooling payoff. Thus, pooling cannot be an equilibrium when $\phi \psi<\pi_{h}$. That is, when $\phi$ is low, in particular, when the fee $f$ is low, the bank will sell the loans to investors individually, using the skin in the game as a signal.

Now, consider the case when $\phi \psi \geq \pi_{h}$, that is when $\phi$, and in particular the fee $f$, is high. The bank's ex ante payoff from pooling, $V_{P}$, is given by the value of selling all loans at the price of $\beta \psi R$ :

$$
V_{P}=2 \phi \beta \psi R
$$

The bank's ex ante payoff from signaling when the loans are sold separately, $V_{S}$, is computed as the weighted average of the payoff to each of the three possible portfolio types. Letting $\rho_{H}:=\theta^{2}, \rho_{M}:=2 \theta(1-\theta)$ and $\rho_{L}:=(1-\theta)^{2}$, the ex ante payoff from signaling is given by

$$
V_{S}=\rho_{H} 2 \beta \pi_{h} R\left(d^{h}+\phi\left(1-d^{h}\right)\right)+\rho_{M}\left(\beta \pi_{h} R\left(d^{h}+\phi\left(1-d^{h}\right)\right)+\beta \pi_{l} R \phi\right)+\rho_{L} 2 \beta \pi_{l} R \phi
$$

Comparing the two payoffs we find that

$$
V_{P}-V_{S}=2 \theta \pi_{h} \beta R(\phi-1) d^{h}>0
$$


This is not surprising given that signaling is costly. Thus, as long as pooling is feasible and the bank can commit to a selling strategy, the bank will pool to sell its loans. When pooling is not feasible, the bank uses costly signaling. In summary:

Lemma 2. Suppose that the bank sells each loan separately to investors. Then,

1. If $\phi<\frac{\pi_{h}}{\psi}$, then the bank will sell the loans using signaling.

2. If $\phi \geq \frac{\pi_{h}}{\psi}$, then the bank will sell the loans using pooling.

\subsection{Portfolio Sales}

Now we allow the bank to bundle the two loans and sell them as a portfolio. The analysis of portfolio sales follows closely the one above for single loan sales. Let $d^{i}$ denote the fraction of a portfolio of type $i(i=H, M, L)$ that the bank keeps on its books. The maximum prices, that an investor will pay for portfolios of type $H, M$ and $L$ are equal to $2 \beta \pi_{h} R, \beta\left(\pi_{h}+\pi_{l}\right) R$ and $2 \beta \pi_{l} R$, respectively, which correspond to the expected payoffs of these portfolios. To simplify notation, let $\pi_{m}:=(1 / 2)\left(\pi_{h}+\pi_{l}\right)$. The following results can be established. ${ }^{4}$

Lemma 3. Under a separating equilibrium of prices and retention strategies with portfolio loan sales:

$$
\begin{gathered}
p_{L}=\beta \pi_{l} R, \quad p_{M}=\beta \pi_{m} R, \quad p_{H}=\beta \pi_{H} R, \quad d^{L}=0, \\
d^{M}=\hat{d}^{M}:=\frac{\phi\left(\frac{\pi_{m}}{\pi_{l}}-1\right)}{\phi\left(\frac{\pi_{m}}{\pi_{l}}\right)-1},
\end{gathered}
$$

and

$$
d^{H}=\hat{d}^{H}:=\frac{\phi\left(\frac{\pi_{h}}{\pi_{m}}-1\right)+d^{M}(\phi-1)}{\phi\left(\frac{\pi_{h}}{\pi_{m}}\right)-1},
$$

where $\hat{d}^{H}>\hat{d}^{M}$.

\footnotetext{
4 The proofs of Lemma 3 and Lemma 4 are provided in a Supplementary Appendix.
} 
With three types of portfolios, the bank needs two signals to separate them. As stated in equation (1), the skin in the game is decreasing with asset quality. It is also easy to establish that Lemma 2 also applies to portfolio sales as well as sales on individual loans.

Lemma 4. Suppose that the bank can either keep the portfolios on its books or sell them to investors. Then,

1. If $\phi<\frac{\pi_{h}}{\psi}$, then the bank will sell the portfolios to investors using signaling,

2. If $\phi \geq \frac{\pi_{h}}{\psi}$, then the bank will sell the portfolios to investors using pooling.

As for the case of single loan sales, costly signaling is only used when pooling generates a lower expected payoff.

The next result compares Lemma 2 and Lemma 4 to determine whether the bank will sell the loans separately or as a portfolio (proof in the Appendix). It reproduces the result from DeMarzo (2005) that the bank will prefer to sell loans singly to investors rather than as a pool of loans. The intuition is straightforward. When the bank sells the loans singly, there are only two types to be separated. In contrast, when the bank sells them as a pool, there are three portfolio types to be separated. Given that signaling is costly, it is better to sell loans singly.

Proposition 1 (DeMarzo (2005)). Suppose that the bank can sell the loans either singly or as a portfolio. Then, the bank will sell them singly to investors.

This result is an important benchmark. The fact that a bank has multiple loans does not in itself provide an explanation for why banks may prefer to sell portfolios of loans.

\section{Mixed Pooling and Signaling}

DeMarzo (2005) offers an explanation for why banks may sell portfolios of loans. His explanation is that banks can repackage loans into tranches of differing risk categories. By creating a tranche that is risk free, a bank ameliorates the lemons problem for this tranche enabling it to sell loans at a higher average price. As mentioned in the Introduction, this 
option is not feasible in our model because a risk-free tranche cannot be created when the minimum payoff from each loan type is zero.

We offer an alternative explanation for why banks may pool loans and retain a proportion of loans on their books. In particular, we consider a sales strategy that involves a mix of pooling portfolios and signaling. Since there are three portfolio types in our model, we consider the case where a bank pools two of these potential portfolios. The advantage of such a strategy is that the bank will only have to separate two portfolios, the mixed portfolio and the unmixed portfolio, and therefore, will use only one signal.

There are three potential portfolios mixes: a pool of portfolios $\mathrm{H}$ and $\mathrm{M}$, which we label as HM, a pool of portfolios $\mathrm{H}$ and L (HL) and a pool of portfolios M and L (ML). For example, a sales strategy mix ML means that when the bank's portfolio is type H, the bank signals it by using skin in the game and, thus, separates it from the other two types, namely, M and L; But, when its portfolio is type M, it does not use skin in the game as a signal and, thus, does not separate the type $\mathrm{M}$ portfolio from the type $\mathrm{L}$ portfolio.

To calculate the skin in the game required for each mix let

$$
\pi_{i j}:=\frac{\rho_{i} \pi_{i}+\rho_{j} \pi_{j}}{\rho_{i}+\rho_{j}}
$$

denote the conditional probability of the successful outcome if the portfolio mix is $i j$, where $i \in\{H, M\}$ and $j \in\{M, L\}, i \neq j .{ }^{5}$ It follows straightforwardly (since $\psi \gtreqless \pi_{m}$ and $\pi_{H L} \gtreqless \psi$ for $\left.\theta \gtreqless 1 / 2\right)$ that $\pi_{H M}>\pi_{l}, \pi_{h}>\pi_{M L}$ and $\pi_{H L} \gtreqless \pi_{m}$ as $\theta \gtreqless 1 / 2$. The expected discounted value, per share, of the portfolio mix $i j$ is therefore $\beta \pi_{i j} R$.

Since prices are competitive, the sale of the mix $i j$ will yield a payoff to the bank of $\phi \beta \pi_{i j} R$. This is to be compared to a payoff from retaining the higher quality portfolio of $\beta \pi_{i} R$. If the bank can commit to a selling strategy, as discussed in the previous section, then it will prefer to retain the higher quality portfolio than the mix provided $\pi_{i}>\phi \pi_{i j}$.

\footnotetext{
${ }^{5}$ Note there is some abuse of notation here. The $\pi_{i}$ on the right hand side of the formula should be $\pi_{h}$ or $\pi_{m}$ depending on whether the portfolio is $i=H$ or $i=M$ respectively.
} 
Thus, a necessary condition for the mixed portfolio $i j$ to be sold is

$$
\phi \pi_{i j} \geq \pi_{i}
$$

As we have seen in the previous section, signaling requires that the bank retain a fraction of the higher quality asset on its books. For the mixed portfolio HM, this means retaining a fraction of the portfolio HM. For the mixed portfolio ML, it means retaining a fraction of the high quality loan. For the mixed portfolio HL, what is retained depends on the proportion of high quality and low quality loans. If $\theta>1 / 2$, then the mix HL has more of the higher quality loans on average than portfolio $\mathrm{M}$ and the mix HL is the higher quality asset which may be retained as a signal. If $\theta<1 / 2$, then the reverse is true and the portolio $M$ is the higher quality asset that may be retained as a signal. If $\theta=1 / 2$, then there is no need to signal because the mix of portfolios $\mathrm{H}$ and $\mathrm{L}$ is exactly equivalent to portfolio M. Let $d^{i j}$ denote the fraction of the higher quality asset retained on the bank's books to signal the portfolio mix $i j$ and $U_{i j}$ the corresponding expected payoff. It can be checked that

$$
\begin{aligned}
U_{H M} & =2\left(\left(\rho_{H}+\rho_{M}\right)\left(d^{H M}+\phi\left(1-d^{H M}\right)\right) \beta \pi_{H M} R+\rho_{L} \phi \beta \pi_{l} R\right), \\
U_{H L} & = \begin{cases}2\left(\left(\rho_{H}+\rho_{L}\right)\left(d^{H L}+\phi\left(1-d^{H L}\right)\right) \beta \pi_{H L} R+\rho_{M} \phi \beta \pi_{m} R\right) & \text { for } \theta \geqslant \frac{1}{2} \\
2\left(\rho_{M}\left(d^{H L}+\phi\left(1-d^{H L}\right)\right) \beta \pi_{m} R+\left(\rho_{H}+\rho_{L}\right) \phi \beta \pi_{H L} R\right) & \text { for } \theta<\frac{1}{2},\end{cases} \\
U_{M L} & =2\left(\rho_{H}\left(d^{M L}+\phi\left(1-d^{M L}\right)\right) \beta \pi_{h} R+\left(\rho_{M}+\rho_{L}\right) \phi \beta \pi_{M L} R\right) .
\end{aligned}
$$

Then, using exactly the same steps as for the case of single loan sales, it can be shown that the skin in the game is given by $d^{i j}=\hat{d}^{i j}$ where

$$
\hat{d}^{H M}:=\frac{\phi\left(\pi_{H M}-\pi_{l}\right)}{\phi \pi_{H M}-\pi_{l}} ; \quad \hat{d}^{H L}:=\left\{\begin{array}{ll}
\frac{\phi\left(\pi_{H L}-\pi_{m}\right)}{\phi \pi_{H L}-\pi_{m}} & \text { for } \theta \geqslant \frac{1}{2} \\
\frac{\phi\left(\pi_{m}-\pi_{H L}\right)}{\phi \pi_{m}-\pi_{H L}} & \text { for } \theta<\frac{1}{2} ;
\end{array} \quad \hat{d}^{M L}:=\frac{\phi\left(\pi_{h}-\pi_{M L}\right)}{\phi \pi_{h}-\pi_{M L}} .\right.
$$

\subsection{Feasible Sales Strategies of Mixed Pooling and Signaling}

Having established the retention strategies for each portfolio mix, we can turn to consider the feasibility of sale of each portfolio mix. Recall that if $\phi \psi \geq \pi_{h}$, then the bank will prefer to pool and sell the whole portfolio to the market. When $\phi \psi<\pi_{h}$, the bank 
will use signaling and may sell the loans separately or may pool two of the three potential portfolios. In the next proposition, we identify sales strategies that are feasible and in the subsequent theorem (next subsection) we show which among the feasible strategies maximize the bank's payoff.

\section{Proposition 2. [Feasible Mixed Pooling and Signaling Strategies]}

1. A pooled mix of portfolios $H$ and $M$ is feasible when $\frac{\pi_{h}}{\pi_{H M}}<\phi \leqslant \frac{\pi_{h}}{\psi}$.

2. A pooled mix of portfolios $H$ and $L$ is feasible when $\theta \geqslant \frac{1}{2}$ and $\frac{\pi_{h}}{\pi_{H L}}<\phi \leqslant \frac{\pi_{h}}{\psi}$.

3. A pooled mix of portfolios $M$ and $L$ is feasible when $\frac{\pi_{m}}{\pi_{M L}}<\phi \leqslant \frac{\pi_{h}}{\psi}$.

Proposition 2 identifies parameter restrictions such that mixed pooling and signaling strategies are feasible. In comparison with single asset sales, each of these new strategies must satisfy an additional incentive constraint. When the bank pools two of the portfolios together, its payoff must be higher than what it could obtain by keeping the higher quality portfolio in its books.

\subsection{Optimal Sales Strategies}

In comparing single loan sales and sales that use mixed pooling and signaling, the bank's payoff from individuals sales is given by $V_{S}$ in equation (5). Comparing $V_{S}$ with the utilities derived from mixed pooling and signaling and comparing the mixed pooling strategies HL and ML, we obtain the following result:

Theorem 1. [Optimal Sales Strategies]

1. If $\phi<\frac{\pi_{m}}{\pi_{M L}}$, then the bank will sell the loans singly to investors using signaling,

2. If $\frac{\pi_{m}}{\pi_{M L}} \leq \phi<\min \left\{\frac{\pi_{h}}{\psi}, \frac{\pi_{h}}{\pi_{H L}}\right\}$, then the bank will pool portfolios $M$ and $L$,

3. If $\frac{\pi_{h}}{\pi_{H L}} \leq \phi<\frac{\pi_{h}}{\psi}$, then the bank will choose either to pool portfolios $H$ and $L$ or pool portfolios $M$ and $L$. For $\theta \in\left(1 / 2,\left(\pi_{h}+\pi_{l}\right) /\left(\pi_{h}+2 \pi_{l}\right)\right)$, the bank will pool portfolios $H$ and $L$. For larger values of $\theta$, there is a critical $\phi^{c}(\theta) \in\left(\pi_{h} / \pi_{H L}, \pi_{h} / \psi\right)$ such that the bank chooses to pool portfolio $H$ and $L$ for $\phi>\phi^{c}(\theta)$ and chooses to pool portfolios $M$ and $L$ for $\phi<\phi^{c}(\theta)$.

4. If $\phi \geqslant \frac{\pi_{h}}{\psi}$, then the bank will sell the loans to investors using pooling. 
Proposition 2 identifies parameter values such that mixed pooling strategies are feasible. Theorem 1 shows which strategies are most profitable for different parameter values. It says that pooling the mix of portfolios $\mathrm{H}$ and $\mathrm{L}$, or the mix of portfolios $\mathrm{M}$ and $\mathrm{L}$, can be optimal. The intuition is as follows. First compare $\hat{d}^{h}$ from equation (3) with $\hat{d}^{M L}$ from equation (7). It can be checked that $\hat{d}^{M L}<\hat{d}^{h}$ : the skin in the game for the mixed portfolio is lower because it is only required if both loans are of high quality, whereas, if loans are sold separately, the skin in the game is required whenever the bank has a high quality loan. Similarly, for $\theta \geq 1 / 2$, it can be checked that $\hat{d}^{H L}<\hat{d}^{h}$ because the skin in the game for the portfolio HL is only required if both loans are of the same type (for $\theta<1 / 2$, the mixed portfolio HL will not be used because it will be dominated by pooling all portfolios). Furthermore, it can be shown that the mixed portfolio HM is never used because it is always dominated by separate loan sales. In particular, the reduction in the cost of signaling the portfolio HM, relative to the cost of signaling the high-quality asset, is not sufficient to compensate for the decline in the value of the pooled portfolio relative to the value of the high-quality asset. In comparing the two mixes HL with ML, it can be shown that $\hat{d}^{H L}<\hat{d}^{M L}$. However, HL requires a skin in the game whenever both loans are of the same quality, whereas the mixed portfolio ML requires a skin in the game only when both loans are of high quality. Whether the mix HL or the mix ML dominates, will depend on the loan mix. ${ }^{6}$ A larger value of $\phi$ decreases the skin in the game required for both portfolio mixes but this effect can be shown to benefit HL relatively more than ML. ${ }^{7}$ We conclude from Theorem 1 that, for certain parameter values, the mix of portfolios $\mathrm{H}$

\footnotetext{
${ }^{6}$ For $\theta$ close to $1 / 2$, the skin in the game required for the portfolio HL will be small whereas the skin in the game for ML remains non-negligible, and hence, the portfolio HL dominates. For $\theta$ close to 1 , the difference in the skin in the game is smaller but the probability that both loans are low quality becomes smaller faster and again the portfolio HL dominates. For intermediate values of $\theta$, whether HL dominates or not depends on the parameter configuration of $\phi, \pi_{h}$ and $\pi_{l}$.

${ }^{7}$ It is shown in the Appendix that a sufficient condition for HL to dominate ML is $\phi>\phi^{c}$ where
}

$$
\phi^{c}=1+\frac{\left(\pi_{h}-\pi_{l}\right)\left(\sqrt{\pi_{h}\left(\pi_{h}+\pi_{l}\right)}-\pi_{h}\right)}{2 \pi_{h}^{2}} .
$$

It can be checked that $\phi^{c}<2 \pi_{h} /\left(\pi_{h}+\pi_{l}\right)$ and therefore, there is a non-empty set of parameter values $\theta \geq 1 / 2$ and $\pi_{h}>\pi_{l}$ such that $\phi>\phi^{c}$ and $\pi_{h} / \pi_{H L} \leq \phi<\pi_{h} / \psi$. It can be shown that $\phi^{c} \leq$ $(10+7 \sqrt{7}) / 27 \approx 1.0563059$. Since $\phi=f+\beta \psi R$ and $\beta \pi_{l} R>1$, the fee required on sales for this condition to be satisfied is quite low. 
and $\mathrm{L}$ is used, which means that low quality loans may be retained by the bank in line with the observation of Acharya et al. (2009). Averaging across banks, a mix of high and low quality loans will be retained. This is in line with the evidence of Keys et al. (2010).

Theorem 1 also sheds some light on how the use of pooling strategies respond to changes in the fee and the interest rate spread. The fee $f$ is directly related to $\phi$. The return $R$ is a good proxy for the interest spread, given that the size of each loan is equal to 1 . Some recent studies find that both the fee and the spread are countercyclical. For example, Berg et al. (2016) find that higher upfront fees are more likely in periods of higher volatility in profitability, when it is more likely that borrowers will draw down their credit lines, and Walentin (2014) documents the countercyclicality of spreads. Given this observed countercyclicality of spreads and fees, Theorem 1 implies that during times of financial turmoil, e.g., 2007-08 global financial crisis, the use of pooling strategies increases. In particular, an implication of our model is that the within portfolio variability of the distribution of predicted default rates (e.g., fico rates for mortgages) increases with the financial volatility.

This implication is, in principle, empirically testable. Testing this implication would require information not only about the portfolios that banks manage but also about the assets that composed those portfolios. There are many data sets containing ratings for individual loans issued over a number of years and also data sets offering information about CDOs. However, what is required is not just information about the tranches, but also information about the whole portfolios from where the tranches were created. To our knowledge, only Faltin-Traeger and Mayer (2012) use a data set with information about both portfolios and the assets that composed those portfolios. Unfortunately, that data set covers only the period 2005-07 and therefore is not useful for testing the implications of our model because it does not cover the period of the financial crisis.

\section{Multiple Sales Rounds}

So far, we have considered only one round of sales. In reality, banks keep recycling their assets by selling new loans and using the proceeds to offer new ones. In this section, we demonstrate how the analysis might be extended to multiple rounds. 
When we consider multiple rounds we face two related problems: Firstly, there is an 'integer problem' related to the number of new loans and, secondly, there are complications in deriving the composition of subsequent portfolios. We avoid these problems, in this section, by considering the case where the portfolio consists of a continuum of assets. Since sales of individual assets are not feasible, we concentrate on portfolio sales and consider only complete separation of portfolio types. In particular, suppose that in each round the portfolio can only be one of the following three types: (a) all loans low quality (probability $\rho_{L}$ ); (b) all loans high quality (probability $\rho_{H}$ ) and (c) half the loans low quality and half the loans high quality (probability $\rho_{M}$ ). This has a close correspondence to the one-period model we have previously considered, but here we restrict the portfolio types in a very arbitrary way. To simplify the exposition, we assume that there are two sub-periods. The multiple rounds of sales take place during the first sub-period, which is very short (no discounting between rounds). At the end of the second sub-period, all loans mature. The idea we try to capture is that the securitization process is very short relative to the duration of loan contracts. With this setting, the bank faces exactly the same problem in each round (only the size of the portfolio changes) and, therefore, the skin in the game will not vary.

As before, let $d^{i}$ denote the fraction of a portfolio of type $i(i=H, M, L)$ that the bank keeps on its books. Since markets are competitive, both sold and retained loans are priced at their expected value, Thus, the bank's payoff from the first round $V_{i}^{1}$ is given by the value of the retained loans plus the per unit fee $f$ times the value of loans sold, that is,

$$
V_{i}^{1}=d^{i} p_{i}+f\left(1-d^{i}\right) p_{i}
$$

Let

$$
W:=\rho_{H} p_{H} d^{H}+\rho_{L} p_{L} d^{L}+\rho_{M} p_{M} d^{M},
$$

and

$$
Z:=\rho_{H}\left(1-d^{H}\right) p_{H}+\rho_{L}\left(1-d^{L}\right) p_{L}+\rho_{M}\left(1-d^{M}\right) p_{M} .
$$

The term $W$ is the expected value of loans to be retained in the next round and $Z$ is the expected value of loans to be sold in the next round. We will restrict our attention to 
problems that satisfy the restriction $Z<1 .^{8}$ The bank's expected payoff from the second round can be shown to be given by

$$
V_{i}^{2}=\left(1-d^{i}\right) p_{i}(W+f Z)
$$

and the expected payoff from the third round is

$$
V_{i}^{3}=\left(1-d^{i}\right) p_{i}(W+f Z) Z
$$

Then, by induction, we have, for $T \geq 2$

$$
V_{i}^{T}=\left(1-d^{i}\right) p_{i}(W+f Z) Z^{T-2}
$$

Adding the payoffs for all periods, we find that the bank's total expected payoff from portfolio $i$ is equal to

$$
\sum_{t=0}^{\infty}\left(d^{i} p_{i}+\left(1-d^{i}\right) p_{i}\left[f+(W+f Z) Z^{t}\right]\right)=d^{i} p_{i}+\left(1-d^{i}\right) p_{i}\left(f+\frac{W+f Z}{1-Z}\right) .
$$

This expression can be compared with the corresponding expression for single round sales: $d^{i} p_{i}+\left(1-d^{i}\right) p_{i}(f+\beta \psi R)$. The difference between these expressions is that the last term includes future fees and retention rates from subsequent rounds. We can, therefore, follow the same steps as those in the previous section of the paper using these modified payoff functions. Conceptually, the problem is identical but technically it is more complicated since the terms $W$ and $Z$ are functions of $d^{i}$.

In the above example, we have restricted our attention to complete separation strategies. By following the same steps as in the last section, we can extend the analysis to portfolios. In principle, the method is simple, but the derivations can quickly become very complicated, especially as the number of asset types, and consequently portfolio types, increase. This complexity may, in itself, be costly and perhaps suggests an explanation for one of the puzzling questions that Gorton and Metrick (2013) have raised in relation

\footnotetext{
${ }^{8}$ An upper bound for $Z$ is $\beta \psi R$, which is greater than one. Thus the assumption $Z<1$ requires that the skin in the game is significant enough. If $Z \geq 1$, then the value of the bank's sales becomes infinite.
} 
to securitization; in their own words "The choice of loans to pool and sell to the SPV also remains a puzzle. Existing theories cannot address why securitized-loan pools are homogeneous - all credit cards or all prime mortgages, for example." The costs of complexity mean it may better to keep securitized-loan pools homogenous.

\section{An Alternative Commitment Strategy}

For pooling to be credible, some commitment by the bank to a selling strategy is required. So far, we have assumed that the bank can commit to a menu of contracts conditional on sales taking place. In this section, we modify this assumption and suppose the bank simply commits to a menu of contracts with each investor separately. Thus, the bank can make credible agreements to individual investors about the contracts that it will use in future sales but cannot credibly commit to use the same contracts with other investors. We also assume that the bank commits to stand ready to sell all its loans.

We demonstrate that pooling equilibria are also feasible in this case and, in particular, that this form of commitment is stronger than that assumed in Section 2. We are going to concentrate on the case of single sales because it is easier to analyze. It will become clear that a similar argument applies to the case of portfolio sales.

Since we want to show that pooling is feasible, we consider the situation where the bank commits to offer a pooling contract to only one of the investors while using the signaling mechanism to sell the other loan to a another investor. If the bank has a portfolio M, it would wish to sell the low quality loan to the first investor using the pooling contract (at the average price) and sell the high quality loan to the second investor using the retention strategy $d^{h}$. The bank's expected (ex ante) payoff from this second contract is given by:

$$
\left(\rho_{H}+\rho_{M}\right) \beta \pi_{h} R\left(d^{h}+\phi\left(1-d^{h}\right)\right)+\rho_{L} \beta \pi_{l} R \phi
$$

Given that such a strategy involves the bank selling a loan of low quality at the average price when it has portfolio M, investors would only accept the pooling contract if they are convinced that the bank has an incentive to offer the same contract to all other investors. Then, because of the commitment to stand ready to sell all its assets, the bank will not use the strategy, provided that it is no worse off selling its loan at the average price. This 
is true when

$$
\left(\rho_{H}+\rho_{M}\right) \beta \pi_{h} R\left(d^{h}+\phi\left(1-d^{h}\right)\right)+\rho_{L} \beta \pi_{l} R \phi \leq \beta \psi R \phi
$$

With $d^{h}$ given in equation (3) and $\psi=\theta \pi_{h}+(1-\theta) \pi_{l}$, this inequality can be simplified to

$$
\phi \geq \frac{(2-\theta) \pi_{h}-(1-\theta) \pi_{l}}{\pi_{h}}
$$

Inequality (8) says that provided $\phi$ is high enough (equivalently the fee $f$ is high enough), offering the pooling contract to all investors is incentive compatible. Recall from Lemma 2, that the pooling equilibrium exists when $\phi \geq \pi_{h} / \psi$. It is easy to check that

$$
\frac{\pi_{h}}{\psi} \geq \frac{(2-\theta) \pi_{h}-(1-\theta) \pi_{l}}{\pi_{h}}
$$

with equality only when $\theta=1$. Thus, inequality (8) shows that the commitment considered in this section is stronger than the commitment assumed in previous sections (there are some additional parameter values where pooling is feasible) because of the commitment of the bank to sell its whole portfolio, even though it does not commit to offer the same contract to all investors. Some commitment by thee bank is, however, needed because if the bank were completely unable to make any commitments, then pooling would not be credible.

\section{Conclusion}

We have extended the DeMarzo and Duffie (1999) signaling model from single sales of risky assets to portfolio sales. We have identified conditions under which signaling at the portfolio level dominates signaling at the single asset level. It has also been shown that the optimal mix of retained assets can involve both high and low quality loans.

In order to keep the analysis simple, we have assumed that banks hold only two uncorrelated loans, which can be one of two types, high or low quality. We comment briefly on the robustness of the model. If there are more than two loans, then the number of potential portfolios increases and there are more possible mixed portfolios to consider but the analysis is not fundamentally changed. Similarly, if there are more than two types 
of loan, there are more potential portfolios and more qualities to signal but the result that lower quality assets are retained is unlikely to change. Equally, as long as the returns are not perfectly correlated across loan types, the motivation for pooling of portfolios remains and, qualitatively, the nature of our result will be unchanged.

\section{Appendix}

\subsection{Proof of Lemma 1}

Step 1 Any solution that satisfies (IC2) and (IC6) will also satisfy (IC1), (IC3), (IC4) and (IC5).

Proof There are six incentive compatibility constraints.

$$
\begin{aligned}
& 2\left(\beta d^{h} \pi_{h} R+\phi\left(1-d^{h}\right) p_{h}\right) \geq \beta d^{h} \pi_{h} R+\phi\left(1-d^{h}\right) p_{h}+\beta d^{l} \pi_{h} R+\phi\left(1-d^{l}\right) p_{l} \\
& 2\left(\beta d^{h} \pi_{h} R+\phi\left(1-d^{h}\right) p_{h}\right) \geq 2\left(\beta d^{l} \pi_{h} R+\phi\left(1-d^{l}\right) p_{l}\right) \\
& \beta d^{h} \pi_{h} R+\phi\left(1-d^{h}\right) p_{h}+\beta d^{l} \pi_{l} R+\phi\left(1-d^{l}\right) p_{l} \geq \beta d^{h} \pi_{h} R+\beta d^{h} \pi_{l} R+2 \phi\left(1-d^{h}\right) p_{h} \\
& \beta d^{h} \pi_{h} R+\phi\left(1-d^{h}\right) p_{h}+\beta d^{l} \pi_{l} R+\phi\left(1-d^{l}\right) p_{l} \geq \beta d^{l} \pi_{h} R+\beta d^{l} \pi_{l} R+2 \phi\left(1-d^{l}\right) p_{l} \\
& 2\left(\beta d^{l} \pi_{l} R+\phi\left(1-d^{l}\right) p_{l}\right) \geq \beta d^{h} \pi_{l} R+\phi\left(1-d^{h}\right) p_{h}+\beta d^{l} \pi_{l} R+\phi\left(1-d^{l}\right) p_{l} \\
& 2\left(\beta d^{l} \pi_{l} R+\phi\left(1-d^{l}\right) p_{l}\right) \geq 2\left(\beta d^{h} \pi_{l} R+\phi\left(1-d^{h}\right) p_{h}\right)
\end{aligned}
$$

(IC1) states that when the portfolio type is $H$ the bank prefers to sell each loan as type $h$ rather than one loan as type $h$ and the other as type $l$. (IC2) states that when the portfolio type is $H$ the bank prefers to sell each loan as type $h$ rather than selling each loan as type $l$. (IC3) states that when the portfolio type is $M$ the bank prefers to sell the type $h$ loan as type $h$ and the type $l$ loan as type $l$ rather than selling both loans as type $h$. (IC4) states that when the portfolio type is $M$ the bank prefers to sell the type $h$ loan as type $h$ and the type $l$ loan as type $l$ rather 
than selling both loans as type $l$. (IC5) states that when the portfolio type is $L$ the bank prefers to sell each loan as type $l$ rather than one loan as type $h$ and the other as type $l$. (IC6) states that when the portfolio type is $L$ the bank prefers to sell each loan as type $l$ rather than selling each loan as type $h$.

Comparing (IC1) and (IC2) it follows that if (IC2) is satisfied, so is (IC1). Comparing (IC5) and (IC6) it follows that if (IC6) is satisfied, so is (IC5). Subtracting $\beta d^{h} \pi_{h} R+\phi\left(1-d^{h}\right) p_{h}$ from both sides of (IC3) we obtain (IC2). Subtracting $\beta d^{l} \pi_{l} R+\phi\left(1-d^{l}\right) p_{l}$ from both sides of (IC4) we obtain (IC6).

QED

We can combine (IC2) and (IC6) to get

$$
\beta\left(d^{h}-d^{l}\right) \pi_{h} R \geq \phi\left(\left(1-d^{l}\right) p_{l}-\left(1-d^{h}\right) p_{h}\right) \geq \beta\left(d^{h}-d^{l}\right) \pi_{l} R
$$

Step $2 p_{h} \geq p_{l}$.

Proof Given that bank's payoff is increasing in $p_{h}$ and $p_{l}$, in any signaling equilibrium at least one of the two constraints described in Step 1 must bind. This is because we can always increase both in such a way that leaves $\left(1-d^{l}\right) p_{l}-\left(1-d^{h}\right) p_{h}$ constant. If $p_{h}=\beta \pi_{h} R$, then the lemma is trivially satisfied. Suppose that $p_{l}=\beta \pi_{l} R$ and that $\beta \pi_{l} R>p_{h}$ and that (A1) is satisfied. Then set $p_{h}=\beta \pi_{l} R$ clearly increasing the bank's payoff. Given that $\beta \psi R>1$, the second inequality is still satisfied. Increasing $p_{h}$ also relaxes the first constraint and therefore we have a contradiction. QED

\section{Step $3 d^{l}=0$.}

Proof Given that $\pi_{h} R>\pi_{l} R$, (A1) implies that $d^{h}>d^{l}$. Further, notice that if a signaling equilibrium exists, Step 2 implies that the bank's payoff will be decreasing in $d^{h}$ and $d^{l}$. Suppose that the first constraint is not binding. Then decrease $d^{h}$ and $d^{l}$ by the same amount so that either $d^{l}=0$ or the first constraint binds. Suppose that the second constraint is not binding. Then reduce $d^{h}$ and $d^{l}$ so that $\left(1-d^{l}\right) p_{l}-\left(1-d^{h}\right) p_{h}$ stays constant, so that either $d^{l}=0$ or the second constraint binds. Then the lemma follows from the fact that at least one of the constraints is not binding.

QED 
Step 3 and (A1) imply that

$$
\beta d^{h} \pi_{h} R \geq \phi\left(p_{l}-\left(1-d^{h}\right) p_{h}\right) \geq \beta d^{h} \pi_{l} R
$$

Step $4 p_{l}=\beta \pi_{l} R$.

Proof Suppose not. Increasing $p_{l}$ relaxes the second constraint in (A2). Before we have argued that if $p_{l}<\beta \pi_{l} R$, then it must be the case that $p_{h}=\beta \pi_{h} R$. Suppose that the first constraint binds. Then increase $p_{l}$ and decrease $d^{h}$ so that the constraint remains binding. This is possible because reducing $d^{h}$ relaxes the constraint and because (A2) implies that $d^{h}>0$. We have a contradiction.

QED

Step 5 In a separating equilibrium the second constraint binds.

$$
\phi\left(p_{l}-\left(1-d^{h}\right) p_{h}\right)=\beta d^{h} \pi_{l} R
$$

Proof This follows from the fact that the payoff is increasing in $p_{h}$ and decreasing in $d^{h}$ and that reducing $d^{h}$ relaxes the first constraint in (A2).

QED

Step $6 p_{h}=\beta \pi_{h} R$.

Proof Solving (A3) for $p_{h}$ we get

$$
p_{h}=\left(\frac{1-\frac{1}{\phi} d^{h}}{1-d^{h}}\right) \beta \pi_{l} R .
$$

Changes in $p_{h}$ and $d^{h}$ affect the bank's payoff only when it sells a loan of type $h$. Substituting the above expression in that payoff we obtain $\beta d^{h} \pi_{h} R+\beta \pi_{l} R\left((\phi-1) d^{h}\right)$ which is increasing in $d^{h}$. Then the proof follows from $\mathrm{d} p_{h} / \mathrm{d}\left(d^{h}\right)>0$.

QED

Setting $p_{h}=\beta \pi_{h} R$ in (A3) and solving for $d^{h}$ completes the proof of the lemma. QED

\subsection{Proof of Proposition 1}

For the case when $\phi<\pi_{h} / \psi$ we compare the two sales strategies for each portfolio type separately. 
a) Type $L$ portfolio.

The bank is indifferent between selling the loans separately or as a portfolio given that in both cases its payoff will be equal to $\phi \beta \pi_{l} R$.

b) Type $M$ portfolio.

The bank's payoff from selling the loans separately is equal to

$$
\beta d^{h} \pi_{h} R+\phi\left(1-d^{h}\right) \beta \pi_{h} R+\phi \beta \pi_{l} R=-(\phi-1) \pi_{h} R d^{h}+\phi\left(\pi_{h}+\pi_{l}\right) R
$$

and its payoff from selling them as portfolio is equal to

$$
\beta d^{M}\left(\pi_{h}+\pi_{l}\right) R+\phi\left(1-d^{M}\right) \beta\left(\pi_{h}+\pi_{l}\right) R=-(\phi-1)\left(\pi_{h}+\pi_{l}\right) R d^{M}+\phi\left(\pi_{h}+\pi_{l}\right) R .
$$

Comparing the two payoffs we find that the bank will sell them separately if

$$
\pi_{h} R d^{h}-\left(\pi_{h}+\pi_{l}\right) R d^{M}<0
$$

Substituting the solution for $d^{h}$ from equation (3) and the solution for $d^{M}$ from the statement of Lemma 3, it follows that

$$
\pi_{h} R d^{h}-\left(\pi_{h}+\pi_{l}\right) R d^{M}=-\frac{\phi \pi_{l}\left(\pi_{h}-\pi_{l}\right)^{2}}{\left(\phi \pi_{h}-\pi_{l}\right)\left(\left(\phi \pi_{h}-\pi_{l}\right)+(\phi-1) \pi_{l}\right)}<0 .
$$

Therefore, the bank will sell separately the two loans.

c) Type $H$ portfolio.

The bank's payoff from selling the loans separately is equal to

$$
2\left(\beta d^{h} \pi_{h} R+\phi\left(1-d^{h}\right) \beta \pi_{h} R\right)=2\left(-(\phi-1) \pi_{h} R d^{h}+\phi \pi_{h} R\right)
$$

and its payoff from selling them as portfolio is equal to

$$
2\left(\beta d^{H} \pi_{h} R+\phi\left(1-d^{H}\right) \beta \pi_{h} R\right)=2\left(-(\phi-1) \pi_{h} R d^{H}+\phi \pi_{h} R\right)
$$


Clearly, the bank will sell them separately if $d^{H}-d^{h}>0$. After some simple algebraic manipulation:

$$
d^{H}-d^{h}=(\phi-1) \frac{\phi \pi_{l}\left(\pi_{h}-\pi_{m}\right)\left(\pi_{m}-\pi_{l}\right)}{\left(\phi \pi_{h}-\pi_{m}\right)\left(\phi \pi_{h}-\pi_{l}\right)\left(\phi \pi_{m}-\pi_{l}\right)},
$$

which is positive because $\pi_{h}>\pi_{m}>\pi_{l}$ and $\phi>1$. Therefore, the bank will sell the two loans separately using signaling.

Lastly, the proof of the second part of the proposition follows from Lemma 2 and Lemma 4.

QED

\subsection{Proof of Proposition 2}

1. $\pi_{H M}>\psi$ which implies that $\pi_{h} / \pi_{H M}<\pi_{h} / \psi$.

2. $\pi_{H L} \geq \psi$ if and only if $\theta \geq 1 / 2$, so that $\pi_{h} / \pi_{H L}<\pi_{h} / \psi$ if and only if $\theta \geq 1 / 2$.

3. We need to compare $\pi_{m} / \pi_{M L}$ and $\pi_{h} / \psi$. It can be shown that $\frac{\pi_{m}}{\pi_{M L}}-\frac{\pi_{h}}{\psi}=$ $-\frac{\left(\pi_{h}-\pi_{l}\right)(1-\theta) \psi}{2 \psi\left(\theta \pi_{h}+\pi_{l}\right)}<0$.Thus, $\pi_{m} / \pi_{M L}<\pi_{h} / \psi$.

Then, the result follows from $\phi \pi_{i j} \geq \pi_{i}$, which is the necessary condition for the mixed portfolio $i j$ to be sold.

QED

\subsection{Proof of Theorem 1}

First, comparing $V_{S}$, given in equation (5), with $U_{H M}$, given in equation (6), and substituting for the conditional probability $\pi_{H M}$, we have ${ }^{9}$

$$
V_{S}-U_{H M}=(\phi-1) \beta R\left(\left(2 \rho_{H}+\rho_{M}\right) \pi_{h}\left(d^{H M}-d^{h}\right)+\rho_{M} \pi_{l} d^{H M}\right) .
$$

It is easily checked that $d^{h}>d^{H M}$. This is intuitive because the skin in the game must be larger to signal a higher quality asset. In comparing the payoffs $V_{S}$ and $U_{H M}$ therefore, there are two effects. The mixed portfolio has the benefit of using a lower skin in the game whenever one or both loans are of high quality. Thus, the first term in the brackets above, $\left(2 \rho_{H}+\rho_{M}\right) \pi_{h}\left(d^{H M}-d^{h}\right)$, is negative. However, the mixed portfolio also

\footnotetext{
${ }^{9}$ For more detailed derivations please consult the Supplementary Appendix.
} 
has a cost if one of the loans is low quality, because the skin in the game $d^{H M}$ is still required for the mixed pooling strategy where no skin in the game is required if loans are completely separated. It can be shown that the latter effect dominates. That is, $\rho_{M} \pi_{l} d^{H M}>\left(2 \rho_{H}+\rho_{M}\right) \pi_{h}\left(d^{h}-d^{H M}\right)$. Substituting for for $d^{H M}$ from equation (7) and for $d^{h}$ from equation (3) (and for the probability $\pi_{H M}$ and $\pi_{m}$ ) into equation (A.4) gives

$$
V_{S}-U_{H M}=(\phi-1) \beta R\left(\frac{\phi\left(\pi_{h}-\pi_{l}\right)^{2} \rho_{M} \pi_{l}\left(2 \rho_{H}+\rho_{M}\right)}{\left(\phi \pi_{h}-\pi_{l}\right)\left(\left(2 \rho_{H}+\rho_{M}\right)\left(\phi \pi_{h}-\pi_{l}\right)+\rho_{M} \pi_{l}(\phi-1)\right)}\right) .
$$

Since $\phi>1$ and $\pi_{h}>\pi_{l}>0$ and $\rho_{M}>0$, it follows that $V_{S}>U_{H M}$. Thus, the mixed HM portfolio is always dominated by signaling.

Similarly, in comparing $U_{M L}$ and $V_{S}$, the skin in the game for the mixed portfolio is only required if both loans are of high quality and the skin in the game $d^{M L}$ is less than the skin in the game, $d^{h}$, required to signal the high quality when loans are signaled separately. Thus, we have

$$
U_{M L}-V_{S}=(\phi-1) \beta R \pi_{h}\left(2 \rho_{H}\left(d^{h}-d^{M L}\right)+\rho_{M} d^{h}\right)
$$

and since $d^{h}>d^{M L}>0$ (which follows from $\pi_{h}>\pi_{M L}>\pi_{l}$ ), $\phi>1$ we have $U_{M L}>V_{S}$ and the mixed portfolio ML will be preferred to signaling loans separately if it is feasible.

When $\theta<1 / 2$, pooling all three portfolios dominates the mixed portfolio HL. Therefore in comparing the mixed portfolio HL with other strategies, we only need to consider the case $\theta \geq 1 / 2$. For $\theta \geq 1 / 2$, the mixed portfolio HL retains the skin in the game, $d^{H L}$, whenever both loans are of the high quality or both are of the low quality. The benefit of this strategy relative to the separating strategy is given by

$$
\begin{aligned}
U_{H L}-V_{S} & =(\phi-1) \beta R\left(\pi_{h}\left(2 \rho_{H}+\rho_{M}\right) d^{h}-\left(2 \pi_{h} \rho_{H}+\pi_{l} \rho_{L}\right) d^{H L}\right) \\
& =(\phi-1) \beta R\left(\pi_{h}\left(2 \rho_{H}+\rho_{M}\right)\left(d^{h}-d^{H L}\right)+\left(\pi_{h} \rho_{M}-\pi_{l} \rho_{L}\right) d^{H L}\right) .
\end{aligned}
$$

Given that $d^{H L}<d^{h}$ (which follows from the inequalities $\pi_{h}>\pi_{H L}, \pi_{m}>\pi_{l}$ and $\phi>1$ ), and $\rho_{M} \geq \rho_{L}$ for $\theta>1 / 2$ (and $\pi_{h}>\pi_{l}$ ), the above expression is always positive. Thus, the mixed portfolio HL will be preferred to signaling loans separately if it is feasible. 
Finally, we compare the payoffs from the mixed portfolios HL and ML. The mixed portfolio HL requires a skin in the game, $d^{H L}$, whenever both loans are of the same quality. In contrast the mixed portfolio ML requires a skin in the game, $d^{M L}$, only when both loans are of high quality. However, the skin in the game required for the mixed portfolio HL is lower than for the mixed portfolio ML: $d^{H L}<d^{M L}$ (this follows from the inequalities $\pi_{h}>\pi_{H L}, \pi_{m}>\pi_{L M}$ and $\phi>1$ ). Thus, we have

$$
U_{H L}-U_{M L}=(\phi-1) \beta R\left(2 \pi_{h} \rho_{H}\left(d^{M L}-d^{H L}\right)-2 \pi_{l} \rho_{L} d^{H L}\right) .
$$

For $\theta=1 / 2, d^{H L}=0$, whereas $d^{M L}>0$. Therefore for $\theta=1 / 2$, the difference $U_{H L}-$ $U_{M L}>0$ and by continuity there is a $\tilde{\theta}$ such that for $\theta \in[1 / 2, \tilde{\theta})$ the difference is strictly positive. In the limit as $\theta \rightarrow 1, \lim _{\theta \rightarrow 1} d^{H L}>0$ whereas $\lim _{\theta \rightarrow 1}\left(d^{M L}-d^{H L}\right)=0$. Since $\rho_{L} \rightarrow 0$ as $\theta \rightarrow 1$, the difference $U_{H L}-U_{M L} \rightarrow 0$ as $\theta \rightarrow 1$. However, it can be checked that the term $\rho_{H}\left(d^{M L}-d^{H L}\right)$ is declining in the limit whereas the term $\rho_{L} d^{H L}$ is nether increasing nor decreasing in the limit as $\theta \rightarrow 1$. Thus, by continuity, there is a range of $\theta,(\hat{\theta}, 1)$ where the difference $U_{H L}-U_{M L}>0$. We can conclude that there is a range of $\theta$ where the mixed HL portfolio dominates the ML portfolio. This range may be the interval $[1 / 2,1)$ or there may be some values of $\theta$ interior to this interval where the ML portfolio dominates.

The skin in the game, for both mixed portfolios ML and HL, is decreasing in $\phi$ and $\lim _{\phi \rightarrow 1} d^{H L} \rightarrow 1$ and $\lim _{\phi \rightarrow 1} d^{M L} \rightarrow 1$ for $\theta \in(1 / 2,1)\left(d^{H L}=0\right.$ for $\left.\theta=1 / 2\right)$. Thus, the term in brackets in equation (A.5) is decreasing in $\phi$ and is negative in the limit as $\phi \rightarrow 1$ for $\theta \in(1 / 2,1)$. Therefore, it is possible to find a critical $\phi^{c}(\theta)$ such that the bracketed term is positive for $\phi>\phi^{c}(\theta)$. It can be shown that $\phi^{c}(\theta)<\pi_{h} / \pi_{H L}$ for $1 / 2 \leq \theta<$ $\left(\pi_{h}+\pi_{l}\right) /\left(\pi_{h}+2 \pi_{l}\right)$ and that $\pi^{c}(\theta) \in\left(\pi_{h} / \pi_{H L}, \pi_{h} / \psi\right)$ for $1>\theta>\left(\pi_{h}+\pi_{l}\right) /\left(\pi_{h}+2 \pi_{l}\right)$. Defining $\phi^{c}:=\max _{\theta} \phi^{c}(\theta)$, it can be shown that

$$
\phi^{c}=1+\frac{\left(\pi_{h}-\pi_{l}\right)\left(\sqrt{\pi_{h}\left(\pi_{h}+\pi_{l}\right)}-\pi_{h}\right)}{2 \pi_{h}^{2}} .
$$

It is clear that since $\pi_{h}>\pi_{l}, \phi^{c} \geq 1$ with equality only if $\pi_{l}=0$. For $\phi>\phi^{c}$ the difference in (A.5) is positive and the mixed portfolio HL will dominate the mixed portfolio ML for any $\theta \in[1 / 2,1)$. It is also easily checked that $\phi^{c}<2 \pi_{h} /\left(\pi_{h}+\pi_{1}\right)$. Since $\pi_{h} / \psi$ and $\pi_{h} / \pi_{H L}$ 
are decreasing in $\theta$ and $\pi_{h} / \psi>\pi_{h} / \pi_{H L}$ for $\theta>1 / 2$ with $\pi_{h} / \psi=\pi_{h} / \pi_{H L}=2 \pi_{h} /\left(\pi_{h}+\pi_{1}\right)$ for $\theta=1 / 2$, it follows that there exist values of $\theta, \pi_{h}$ and $\pi_{l}$ such that $\phi>\phi^{c}$ and $\pi_{h} / \pi_{H L} \leq \phi<\pi_{h} / \psi$.

To complete the proof we note that the inequalities $\pi_{h}>\pi_{m}$ and $\pi_{H L}<\pi_{M L}$ imply that $\frac{\pi_{m}}{\pi_{M L}}<\frac{\pi_{h}}{\pi_{H L}}$. Hence, considering each of the statements of the theorem in turn:

1. Follows from $\pi_{m} / \pi_{M L}<\pi_{h} / \pi_{H L}$ and the the necessary conditions $\phi \pi_{M L} \geq \pi_{m}$ and $\phi \pi_{H L} \geq \pi_{h}$, that neither the mixed portfolio ML nor the mixed portfolio HL will be sold for $\phi<\pi_{m} / \pi_{M L}$. Neither will the mixed portfolio HM be sold because, as we have shown above, it is dominated by signaling of separate loan sales, $V_{S}>U_{H M}$.

2. For $\theta<1 / 2, \pi_{h} / \psi<\pi_{h} / \pi_{H L}$ and therefore the condition $\phi \pi_{H L} \geq \pi_{h}$ for the mixed portfolio HL to be sold is not satisfied. The mixed portfolio ML may be sold because $\phi \geq \pi_{m} / \pi_{M L}$ and since $U_{M L}>V_{S}$, this portfolio dominates separate loan sales, which in turn dominates the mixed portfolio HM.

3. Since $\pi_{m} / \pi_{M L}<\pi_{h} / \pi_{H L} \leq \phi$, the necessary conditions for the sale of the mixed portfolios ML and HL are both satisfied. Since $\phi<\pi_{h} / \psi$, pooling does not dominate these mixed portfolios. Since $U_{M L}>V_{S}$ and $U_{H L}>V_{S}$, both mixed portfolios are better than signaling loans separately. The comparison of the two mixed portfolios for $\theta \geq 1 / 2$ depends on the sign of $U_{H L}-U_{M L}$, which as shown above is positive for $\theta$ near to one and $\theta$ near to $1 / 2$ and is such that for $\phi>\phi^{c}$, the mixed portfolio HL dominates for all $\theta \in[1 / 2,1)$.

4. Follows from the domination of the pooling strategy when $\phi \geq \pi_{h} / \psi$.

QED

\section{References}

Viral V. Acharya, Thomas Philippon, Matthew Richardson, and Nouriel Roubini. The financial crisis of 2007-2009: Causes and remedies. In Viral V. Acharya and Matthew Richardson, editors, Restoring Financial Stability: How to Repair a Failed System, pages 1-56. Wiley, 2009. ISBN 978-0-470-49934-4. 
Tobias Berg, Anthony Saunders, and Sascha Steffen. The total cost of corporate borrowing in the loan market: Don't ignore the fees. Journal of Finance, 71(3):1357-1392, May 2016. doi: 10.1111/jofi.12281.

Weitzu Chen, Chi-Chun Liu, and Stephen G. Ryan. Characteristics of securitizations that determine issuers' retention of the risks of the securitized assets. The Accounting Review, 83(5):1181-1215, September 2008. doi: 10.2308/accr.2008.83.5.1181.

Peter DeMarzo. The pooling and tranching of securities: A model of informed intermediation. Review of Financial Studies, 18(1):1-35, March 2005. doi: 10.1093/rfs/hhi008.

Peter DeMarzo and Darrell Duffie. A liquidity-based model of security design. Econometrica, 67(1):65-100, January 1999. doi: 10.1111/1468-0262.00004.

Cem Demiroglu and Christopher James. How important is having skin in the game? Originator-sponsor affiliation and losses on mortgage-backed securities. Review of Financial Studies, 25(11):3217-3258, November 2012. doi: 10.1093/rfs/hhs095.

Oliver Faltin-Traeger and Christopher Mayer. Lemons and CDOs: Why did so many lenders issue poorly performing CDOs? Working Paper, Columbia Business School, May 2012.

Gary Gorton and Andrew Metrick. Securitization. In G. Constantinides, M. Harris, and R. Stulz, editors, Handbook of the Economics of Finance, volume 2, chapter 1, pages 1-70. Elsevier, 2013. ISBN 978-0-444-53594-8.

Gary B. Gorton and Nicholas S. Souleles. Special purpose vehicles and securitization. In Mark Carey and René M. Stulz, editors, The Risks of Financial Institutions, chapter 12, pages 549-602. University of Chicago Press, 2007. ISBN 978-0-226-09285-0.

Barney Hartman-Glaser. Reputation and signaling in asset sales. Journal of Financial Economics, 125(2):245-265, August 2017. doi: 10.1016/j.jfineco.2017.05.009.

Benjamin J. Keys, Tanmoy Mukherjee, Amit Seru, and Vikrant Vig. Did securitization lead to lax screening? Evidence from subprime loans. The Quarterly Journal of Economics, 125(1):307-362, February 2010. doi: 10.1162/qjec.2010.125.1.307. 
Hayne Leland and David H. Pyle. Informational asymmetries, financial structure, and financial intermediation. Journal of Finance, 32(2):371-387, May 1977. doi: $10.2307 / 2326770$.

Henri Pagès. Bank monitoring incentives and optimal ABS. Journal of Financial Intermediation, 22(1):30-54, January 2013. doi: 10.1016/j.jfi.2012.06.001.

Andrei Shleifer and Robert Vishny. Unstable banking. Journal of Financial Economics, 97(3):306-318, September 2010. doi: 10.1016/j.jfineco.2009.10.007.

Karl Walentin. Business cycle implications of mortgage spreads. Journal of Monetary Economics, 67(C):62-77, October 2014. doi: 10.1016/j.jmoneco.2014.07.005.

\section{Supplementary Appendix (not intended for publication)}

\subsection{Portfolio Sales}

Let $2 p_{i}$ equal the price that the bank is willing to sell a portfolio of type $i$ (that is $p_{i}$ denotes half the portfolio price). Investor participation requires that

$$
p_{H} \leqslant \beta \pi_{h} R, p_{M} \leqslant \beta \pi_{m} R \equiv \beta \pi_{M} R \text { and } p_{L} \leqslant \beta \pi_{l} R
$$

Suppose that the bank's portfolio type is type $H$. Under a signaling equilibrium, the bank's expected payoff from the sale of its portfolio is equal to $2\left(\beta d^{H} \pi_{h} R+\phi\left(1-d^{H}\right) p_{H}\right)$. The interpretation is similar to that for the case for single loan sales. Similar arguments show that the bank's expected payoff when its portfolio is type $M$ is equal to $2\left(\beta d^{M} \pi_{m} R+\phi\left(1-d^{M}\right) p_{M}\right)$ and its expected payoff when its portfolio is type $L$ is equal to $2\left(\beta d^{L} \pi_{l} R+\phi\left(1-d^{L}\right) p_{L}\right)$.

The bank will prefer to sell a fraction of a type $i$ portfolio to investors rather than keeping it on its books if the following condition is satisfied:

$$
\beta d^{i} \pi_{i} R+\phi\left(1-d^{i}\right) p_{i} \geq \beta \pi_{i} R
$$


or

$$
\phi p_{i} \geq \beta \pi_{i} R
$$

where $\pi_{H}=\pi_{h}, \pi_{M}=\frac{\pi_{h}+\pi_{l}}{2}$ and $\pi_{L}=\pi_{l}$.

For signaling to be effective the following incentive compatibility constraints must also be satisfied:

$$
\begin{gathered}
\beta d^{H} \pi_{h} R+\phi\left(1-d^{H}\right) p_{H} \geq \beta d^{M} \pi_{h} R+\phi\left(1-d^{M}\right) p_{M} \\
\beta d^{H} \pi_{h} R+\phi\left(1-d^{H}\right) p_{H} \geq \beta d^{L} \pi_{h} R+\phi\left(1-d^{L}\right) p_{L} \\
\beta d^{M} \pi_{m} R+\phi\left(1-d^{M}\right) p_{M} \geq \beta d^{H} \pi_{m} R+\phi\left(1-d^{H}\right) p_{H} \\
\beta d^{M} \pi_{m} R+\phi\left(1-d^{M}\right) p_{M} \geq \beta d^{L} \pi_{m} R+\phi\left(1-d^{L}\right) p_{L} \\
\beta d^{L} \pi_{l} R+\phi\left(1-d^{L}\right) p_{L} \geq \beta d^{M} \pi_{l} R+\phi\left(1-d^{M}\right) p_{M} \\
\beta d^{L} \pi_{l} R+\phi\left(1-d^{L}\right) p_{L} \geq \beta d^{H} \pi_{l} R+\phi\left(1-d^{H}\right) p_{H}
\end{gathered}
$$

Each of the above expressions is equal to half the expected payoff of the corresponding portfolio. (SIC1) states that when the portfolio type is $H$ the bank prefers to sell it as type $H$ rather than selling it as type $M$. (SIC2) states that when the portfolio type is $H$ the bank prefers to sell it as type $H$ rather than selling it as type $L$. (SIC3) states that when the portfolio type is $M$ the bank prefers to sell it as type $M$ rather than selling it as type $H$. (SIC4) states that when the portfolio type is $M$ the bank prefers to sell it as type $M$ rather than selling it as type $L$. (SIC5) states that when the portfolio type is $L$ the bank prefers to sell it as type $L$ rather than selling it as type $M$. (SIC6) states that when the portfolio type is $L$ the bank prefers to sell it as type $L$ rather than selling it as type $H$.

The constraints can be written as:

$$
\begin{gathered}
\beta \pi_{h} R\left(d^{H}-d^{M}\right) \geq \phi\left(\left(1-d^{M}\right) p_{M}-\left(1-d^{H}\right) p_{H}\right) \\
\beta \pi_{h} R\left(d^{H}-d^{L}\right) \geq \phi\left(\left(1-d^{L}\right) p_{L}-\left(1-d^{H}\right) p_{H}\right) \\
\beta \pi_{m} R\left(d^{H}-d^{M}\right) \leqslant \phi\left(\left(1-d^{M}\right) p_{M}-\left(1-d^{H}\right) p_{H}\right) \\
\beta \pi_{m} R\left(d^{M}-d^{L}\right) \geq \phi\left(\left(1-d^{L}\right) p_{L}-\left(1-d^{M}\right) p_{M}\right)
\end{gathered}
$$




$$
\begin{aligned}
& \beta \pi_{l} R\left(d^{M}-d^{L}\right) \leqslant \phi\left(\left(1-d^{L}\right) p_{L}-\left(1-d^{M}\right) p_{M}\right) \\
& \beta \pi_{l} R\left(d^{H}-d^{L}\right) \leqslant \phi\left(\left(1-d^{L}\right) p_{L}-\left(1-d^{H}\right) p_{H}\right)
\end{aligned}
$$

We can now prove Lemma 3 and Lemma 4.

\subsubsection{Proof of Lemma 3}

Step S1 $d^{H}>d^{M}>d^{L}$.

Proof The first inequality follows from (SIC1*) and (SIC3*). The second inequality follows from $\left(\mathrm{SIC}^{*}\right)$ and $\left(\mathrm{SIC} 5^{*}\right)$. Notice that $\left(\mathrm{SIC} 2^{*}\right)$ and $(\mathrm{SIC} 6 *)$ also imply that $d^{H}>d^{L}$.

QED

Step S2 Any solution that satisfies (SIC1*), $\left(\mathrm{SIC} 3^{*}\right),\left(\mathrm{SIC} 4^{*}\right)$ and $\left(\mathrm{SIC} 5^{*}\right)$ will also satisfy $\left(\mathrm{SIC}^{*}\right)$ and $\left(\mathrm{SIC}^{*}\right)$.

\section{Proof}

$$
\begin{gathered}
\beta \pi_{h} R\left(d^{H}-d^{L}\right) \geq \beta \pi_{h} R\left(d^{H}-d^{M}\right)+\beta \pi_{m} R\left(d^{M}-d^{L}\right) \geq \\
\phi\left(\left(1-d^{M}\right) p_{M}-\left(1-d^{H}\right) p_{H}+\left(1-d^{L}\right) p_{L}-\left(1-d^{M}\right) p_{M}\right)= \\
\phi\left(\left(1-d^{L}\right) p_{L}-\left(1-d^{H}\right) p_{H}\right)
\end{gathered}
$$

The second weak inequality follows from adding (SIC1*) and (SIC4*).

$$
\begin{gathered}
\beta \pi_{l} R\left(d^{H}-d^{L}\right) \leqslant \beta \pi_{m} R\left(d^{H}-d^{M}\right)+\beta \pi_{l} R\left(d^{M}-d^{L}\right) \leqslant \\
\phi\left(\left(1-d^{M}\right) p_{M}-\left(1-d^{H}\right) p_{H}+\left(1-d^{L}\right) p_{L}-\left(1-d^{M}\right) p_{M}\right)= \\
\phi\left(\left(1-d^{L}\right) p_{L}-\left(1-d^{H}\right) p_{H}\right)
\end{gathered}
$$

The second weak inequality follows from adding (SIC3*) and (SIC5*).

We can combine (SIC1*) and (SIC3*) to get

$$
\beta \pi_{h} R\left(d^{H}-d^{M}\right) \geq \phi\left(\left(1-d^{M}\right) p_{M}-\left(1-d^{H}\right) p_{H}\right) \geq \beta \pi_{m} R\left(d^{H}-d^{M}\right)
$$


We can combine $\left(\mathrm{SIC} 4^{*}\right)$ and $\left(\mathrm{SIC} 5^{*}\right)$ to get

$$
\beta \pi_{m} R\left(d^{M}-d^{L}\right) \geq \phi\left(\left(1-d^{L}\right) p_{L}-\left(1-d^{M}\right) p_{M}\right) \geq \beta \pi_{l} R\left(d^{M}-d^{L}\right)
$$

Step S3 $p_{L} \leqslant p_{M} \leqslant p_{H}$.

\section{Proof}

a) Suppose that $p_{L}>p_{M}$. At least one of the following is true: the first constraint in (S4) binds or $p_{L}=\beta \pi_{l} R$.

We first show that in both cases the fist constraint in (S3) must bind. Suppose that $p_{L}=\beta \pi_{l} R$. Then $p_{M}<\beta \pi_{l} R$ implies that the second constraint in (S4) does not bind (given that it does not bind for $p_{L}=p_{M}=\beta \pi_{l} R$ ). Given that bank's payoff is increasing in $p_{M}$ the first constraint in (S3) must bind. Next, suppose that $p_{L}<\beta \pi_{l} R$. Then the first constraint in (S4) binds which implies that the second constraint does not bind and, as before, it must be the case that the first constraint in (S3) binds.

Decrease $d^{M}$ and $p_{M}$ so that the bank's payoff $\beta d^{M} \pi_{m} R+\phi\left(1-d^{M}\right) p_{M}$ remains constant. Notice that Step S1 implies that $d^{M}>0$, and that if $p_{M}=0$, the first constraint in (S3) is not satisfied. Totally differentiating and rearranging we find that the changes must satisfy $\frac{\mathrm{d} p_{M}}{\mathrm{~d}\left(d^{M}\right)}=\frac{\phi p_{M}-\beta \pi_{m} R}{\phi\left(1-d^{M}\right)}$ where the numerator must be positive for the bank to be willing to sell a portfolio of type $M$. The change does not affect (S4) but relaxes the first constraint in (S3). Therefore, we have a contradiction.

b) Suppose that $p_{M}>p_{H}$. The inequality $p_{H}<\beta \pi_{h} R$ implies that the second constraint in (S3) binds. It must also be true that $p_{M}<\beta \pi_{m} R$ (given it does not bind for $p_{H}=p_{M}=\beta \pi_{m} R$ ). But then it follows that the second constraint in (S4) must bind (if not increase $p_{M}$, thus, raising the bank's payoff). Increase $d^{M}$ and $p_{M}$ so that the second constraint still binds. But given that $\pi_{m} R>\pi_{l} R$ the change relaxes the second constraint in (S3) and also increases the bank's payoff. Therefore, we have a contradiction.

QED

Step S4 $d^{L}=0$. 
Proof Suppose that the first constraint in (S4) does not bind. Then decrease $d^{M}$ and $d^{L}$ by the same amount so that either $d^{L}=0$ or the first constraint binds. Suppose that the second constraint is not binding. Then reduce $d^{M}$ and $d^{L}$ so that $\left(1-d^{L}\right) p_{L}-$ $\left(1-d^{M}\right) p_{M}$ stays constant so that either $d^{L}=0$ or the second constraint binds. Then, as long as the changes have not violated the constraints in (S3), the lemma follows from the fact that at least one of the inequalities is not binding. If one of the constraints in (S3) is violated, then decrease $d^{H}$ either by the same amount as $d^{M}$ when the first constraint is the one that binds or decrease $d^{H}$ so that to keep $\left(1-d^{M}\right) p_{M}-\left(1-d^{H}\right) p_{H}$ constant if the second constraint is the one that binds. QED

Step S5 In a signaling equilibrium the second constraint in (S3) and the second constraint in (S4) bind. Further, $p_{L}=\beta \pi_{l} R$.

Proof Suppose that the second constraint in (S3) does not bind. Then we have $p_{H}=$ $\beta \pi_{h} R$. But then the constraint can be relaxed by decreasing $d^{H}$ and thus increasing the bank's payoff. We have a contradiction. Next, suppose that the second constraint in (S4) does not bind. Then, it must be the case that the first constraint in (S3) binds. If $p_{M}<\beta \pi_{m} R$, then increase $p_{M}$ till either the second constraint binds or $p_{M}=\beta \pi_{m} R$. (This is feasible because the first constraint in (S3) does not bind.) Thus, we have a contradiction. In contrast, if $p_{M}=\beta \pi_{m} R$, decrease $d^{M}$, thus, relaxing the constraint. We also have a contradiction. Given that the second constraint in (S4) binds, we have $p_{L}=\beta \pi_{l} R$.

QED

Then, a signaling equilibrium must satisfy (S2) and the following constraints:

$$
\phi\left(\left(1-d^{M}\right) p_{M}-\left(1-d^{H}\right) p_{H}\right)=\beta \pi_{m} R\left(d^{H}-d^{M}\right)
$$

and

$$
\phi\left(\beta \pi_{l} R-\left(1-d^{M}\right) p_{M}\right)=\beta \pi_{l} R d^{M}
$$

Step S6 $p_{M}=\beta \pi_{m} R, p_{H}=\beta \pi_{h} R$.

\section{Proof}


a) Solve (S5) for $p_{H}$ to get

$$
p_{H}=\frac{1-d^{M}}{1-d^{H}} p_{M}+\frac{\beta}{\phi} \pi_{m} R \frac{d^{H}-d^{M}}{1-d^{H}} .
$$

Changes in $p_{H}$ and $d^{H}$ affect the bank's payoff only when it sells a portfolio of type $H$. Substituting the above expression in that payoff we obtain

$$
\beta d^{H} \pi_{h} R+\phi\left(\left(1-d^{M}\right) p_{M}+\frac{\beta}{\phi} \pi_{m} R\left(d^{H}-d^{M}\right)\right)
$$

which is increasing in $d^{H}$. Then the first part of Step S6 follows from $\frac{\mathrm{d} p_{H}}{\mathrm{~d}\left(d^{H}\right)}>0$.

b) Solve (S6) for $p_{M}$ to get

$$
p_{M}=\beta \pi_{l} R\left(\frac{1}{1-d^{M}}+\frac{1}{\phi} \frac{d^{M}}{1-d^{M}}\right) .
$$

Changes in $p_{M}$ and $d^{M}$ affect the bank's payoff only when it sells a portfolio of type $M$. Substituting the above expression in that payoff we obtain

$$
\beta d^{M} \pi_{m} R+\phi \beta \pi_{l} R\left(1+\frac{1}{\phi} d^{M}\right)
$$

which is increasing in $d^{M}$. Then the second part of Step S6 follows from $\frac{\mathrm{d} p_{M}}{\mathrm{~d}\left(d^{M}\right)}>0$. QED

To complete the proof of the lemma substitute the results of Step S6 in (S5) and (S6). Solve (S6) for $d^{M}$. Then substitute the latter solution in (S5) and solve for $d^{H}$.

$$
d^{H}=\frac{\phi\left(\frac{\pi_{h}}{\pi_{m}}-1\right)+d^{M}(\phi-1)}{\phi \frac{\pi_{h}}{\pi_{m}}-1}
$$

After substituting the solution for $d^{M}$ in the above expression and subtract the denominator from the numerator we find that the difference is equal to $d^{M}-1<0$ and therefore $d^{H}<1$. Lastly, $d^{H}-d^{M}=\frac{\phi\left(\frac{\pi_{h}}{\pi_{m}}-1\right)\left(1-d^{M}\right)}{\frac{\pi_{h}}{\pi_{m}}-1}>0$.

QED. 


\subsubsection{Proof of Lemma 4}

We consider the possibility of pooling equilibria. If a pooling equilibrium exists, then the bank will not keep any fraction of the portfolio on its books. The maximum price that investors would be willing to pay for a portfolio (assuming that the bank is willing to sell all types of portfolios) is equal to $2 \beta \psi R$. If the bank keeps a type $H$ portfolio on its books, its payoff will be equal to $2 \beta \pi_{h} R$. If the bank sells the portfolio to investors, its payoff will be $2 \beta \phi \psi R$. Then, the bank will prefer to keep the portfolio on its books if $\phi<\frac{\pi_{h}}{\psi}$. Clearly, if the bank is willing to sell the type $H$ portfolio, it will also be willing to sell portfolios of types $M$ and $L$. The above argument together with Lemma 3 and (S2) imply that if $\phi<\frac{\pi_{h}}{\psi}$, then the bank will sell the portfolio to investors using the skin in the game as a signal.

Next, we need to compare signaling and pooling when $\phi \geq \frac{\pi_{h}}{\psi}$. The bank's payoff from pooling is equal to ${ }^{10}$

$$
W_{P}=V_{P}=2 \phi \beta \psi R=2 \phi \beta\left(\theta \pi_{h}+(1-\theta) \pi_{l}\right) R
$$

The bank's payoff from signaling when the loans are sold together as a portfolio is equal to

$$
\begin{gathered}
W_{S}=\theta^{2} 2 \beta \pi_{h} R\left(d^{H}+\phi\left(1-d^{H}\right)\right)+ \\
2 \theta(1-\theta) \beta\left(\pi_{h}+\pi_{l}\right) R\left(d^{M}+\phi\left(1-d^{M}\right)\right)+(1-\theta)^{2} 2 \beta \pi_{l} R \phi . \\
W_{S}-V_{P}=-2 \theta \beta \pi_{h} R(\phi-1) d^{H}-\theta(1-\theta) 2(\phi-1) d^{M}<0 .
\end{gathered}
$$

QED.

\subsection{Further Details of Proof of Theorem 1}

In comparing the separate loans and portfolio HM we have (repeating (A.4))

$$
V_{S}-U_{H M}=(\phi-1) \beta R\left(\left(2 \rho_{H}+\rho_{M}\right) \pi_{h}\left(d^{H M}-d^{h}\right)+\rho_{M} \pi_{l} d^{H M}\right) .
$$

\footnotetext{
10 The bank's payoff from pooling does not depend on whether the loans are sold separately or as a portfolio.
} 
Substituting for for $d^{H M}$ from equation (7) and for $d^{h}$ from equation (3) gives

$$
V_{S}-U_{H M}=(\phi-1) \beta R\left(\frac{\phi\left(\pi_{H M}-\pi_{l}\right) \pi_{l} \rho_{M}}{\left(\phi \pi_{H M}-\pi_{l}\right)}-\pi_{h} \frac{\phi\left(\pi_{h}-\pi_{l}\right)}{\left(\phi \pi_{h}-\pi_{l}\right)}+\frac{\phi\left(\pi_{H M}-\pi_{l}\right)}{\left(\phi \pi_{H M}-\pi_{l}\right)}\left(2 \rho_{H}+\rho_{M}\right)\right) .
$$

Substituting for the conditional probabilities $\pi_{i j}$ and $\pi_{m}=(1 / 2)\left(\pi_{h}+\pi_{l}\right)$ gives

$$
V_{S}-U_{H M}=(\phi-1) \beta R\left(\frac{\phi\left(\pi_{h}-\pi_{l}\right)^{2} \rho_{M} \pi_{l}\left(2 \rho_{H}+\rho_{M}\right)}{\left(\phi \pi_{h}-\pi_{l}\right)\left(\left(2 \rho_{H}+\rho_{M}\right)\left(\phi \pi_{h}-\pi_{l}\right)+\rho_{M} \pi_{l}(\phi-1)\right)}\right),
$$

as appears in the proof in the main text.

In comparing $U_{H M}$ and $U_{M L}$ we have

$$
U_{H L}-U_{M L}=(\phi-1) \beta R\left(2 \pi_{h} \rho_{H}\left(d^{M L}-d^{H L}\right)-2 \pi_{l} \rho_{L} d^{H L}\right) .
$$

Substituting for $d^{M L}$ and $d^{H L}$ gives

$$
U_{H L}-U_{M L}=(\phi-1) \beta R \pi_{l} \rho_{L} d^{H L}\left(\left(\frac{\pi_{h} \rho_{H}}{\pi_{l} \rho_{L}}\right)\left(\frac{d^{M L}-d^{H L}}{d^{H L}}\right)-1\right) .
$$

The sign of $U_{H L}-U_{M L}$ depends on the sign of the bracketed term. Substituting for $d^{H L}$ and $d^{M L}$ gives

$$
\left(\frac{\pi_{h} \rho_{H}}{\pi_{l} \rho_{L}}\right)\left(\frac{d^{M L}-d^{H L}}{d^{H L}}\right)-1=\frac{\pi_{h} \rho_{H}(\phi-1)\left(\pi_{h} \pi_{m}-\pi_{H L} \pi_{M L}\right)}{\pi_{l} \rho_{L}\left(\pi_{H L}-\pi_{m}\right)\left(\phi \pi_{h}-\pi_{M L}\right)}-1 .
$$

Differentiating this term with respect to $\phi$ gives the derivative

$$
\frac{\pi_{h} \rho_{H}\left(\pi_{h}-\pi_{M L}\right)\left(\pi_{h} \pi_{m}-\pi_{H L} \pi_{M L}\right)}{\pi_{l} \rho_{L}\left(\pi_{H L}-\pi_{m}\right)\left(\phi \pi_{h}-\pi_{M L}\right)^{2}} .
$$

This is positive because $\pi_{h}>\pi_{M L}, \pi_{H L}>\pi_{m}$ and $\pi_{h} \pi_{m}>\pi_{H L} \pi_{M L}$. Hence, there will a critical value of $\phi, \phi^{c}$ such that $U_{H L} \gtreqless U_{M L}$ and $\phi \gtreqless \phi^{c}$. This critical value of $\phi$ depends on parameters and in particular depends on $\theta$ because $\rho_{i}$ is a function of $\theta$. Hence, we write $\phi^{c}(\theta)$. Solving

$$
\frac{\pi_{h} \rho_{H}(\phi-1)\left(\pi_{h} \pi_{m}-\pi_{H L} \pi_{M L}\right)}{\pi_{l} \rho_{L}\left(\pi_{H L}-\pi_{m}\right)\left(\phi \pi_{h}-\pi_{M L}\right)}-1=0
$$


gives

$\phi^{c}(\theta)=1+\frac{\pi_{l}\left(\pi_{H L}-\pi_{m}\right)\left(\pi_{h}-\pi_{M L}\right) \rho_{L}}{\pi_{h}\left(\pi_{h} \pi_{m}-\pi_{H L} \pi_{M L}\right) \rho_{H}+\pi_{l}\left(\pi_{m}-\pi_{M L}\right) \rho_{L}}=1+\frac{\pi_{l}\left(\pi_{h}-\pi_{l}\right)(1-\theta)(2 \theta-1)}{\pi_{h}\left(\pi_{h} \theta^{2}+\pi_{l}(1-\theta)^{2}\right)}$

where the second equality follows from substituting for the the conditional probabilities and for the probabilities $\rho_{i}$. It follows that $\phi^{c}(\theta)>1$ for $\theta \in(1 / 2,1)$ and $\phi^{c}(1 / 2)=$ $\phi^{c}(1)=1$. We are interested in $\pi_{h} / \pi_{H L} \leq \phi<\pi_{h} / \psi$. We have

$$
\frac{\pi_{h}}{\psi}-\phi^{c}(\theta)=\frac{\pi_{h}}{\pi_{h} \theta+\pi_{l}(1-\theta)}-\phi^{c}(\theta)=\frac{\left(\pi_{h}-\pi_{l}\right)(1-\theta)\left(\pi_{h}\left(\pi_{h}-\pi_{l}\right) \theta^{2}+\pi_{l}(1-\theta)\left(\pi_{h}-\pi_{l}(2 \theta-1)\right)\right.}{\pi_{h}\left(\pi_{h} \theta+\pi_{l}(1-\theta)\right)\left(\pi_{h} \theta^{2}+\pi_{l}(1-\theta)^{2}\right)} .
$$

Since terms on the LHS are positive for $\theta \in[1 / 2,1]$, we have $\phi^{c}(\theta)<\pi_{h} / \psi$ for $\theta \in[1 / 2,1)$. Equally,

$$
\phi^{c}(\theta)-\frac{\pi_{h}}{\pi_{H L}}=\phi^{c}(\theta)-\frac{\theta^{2}+(1-\theta)^{2}}{\pi_{h} \theta^{2}+\pi_{l}(1-\theta)^{2}}=\frac{\left(\pi_{h}-\pi_{l}\right)(1-\theta)\left(\pi_{l}(2 \theta-1)-\pi_{h}(1-\theta)\right)}{\pi_{h} \theta^{2}+\pi_{l}(1-\theta)^{2}} .
$$

The above term has the same sign as the sign of $\pi_{l}(2 \theta-1)-\pi_{h}(1-\theta)$. Thus, we have

$$
\phi^{c}(\theta)-\frac{\pi_{h}}{\pi_{H L}} \gtreqless 0 \quad \text { as } \quad \theta \gtreqless \frac{\pi_{h}+\pi_{l}}{\pi_{h}+2 \pi_{l}} .
$$

It is checked that $2 / 3<\left(\pi_{h}+\pi_{l}\right) /\left(\pi_{h}+2 \pi_{l}\right) \leq 1$ with the second weak inequality holding as equality only if $\pi_{l}=1$. It is possible to find the $\theta^{*}$ that maximizes $\phi^{c}(\theta)$. Solving gives

$$
\theta^{*}=\frac{\left(\pi_{h}-\pi_{l}\right)+\sqrt{\pi_{h}\left(\pi_{h}+\pi_{l}\right)}}{3 \pi_{h}-\pi_{l}}
$$

Substituting into $\phi^{c}(\theta)$ gives

$$
\phi^{c}:=\phi^{c}\left(\theta^{*}\right)=1+\frac{\left(\pi_{h}-\pi_{l}\right)\left(\sqrt{\pi_{h}\left(\pi_{h}+\pi_{l}\right)}-\pi_{h}\right)}{2 \pi_{h}^{2}} .
$$


The maximum value of $\phi^{c}$ occurs when $\pi_{l}=(1 / 9)(2 \sqrt{7}-1) \pi_{h} \approx 0.476834 \pi_{h}$. Hence, substituting into the the formula for $\phi^{c}\left(\theta^{*}\right)$ gives

$$
\phi^{c} \leq \frac{1}{27}(10+7 \sqrt{7}) \approx 1.0563059
$$

